\section{Hypoxia-inducible factor 1-alpha target protein up-regulation in hypoxic cochlear neurons is associated with aged-related hearing loss in C57BL/6 mice}

Emilie Donadieu, 1 Catherine Riva²

1Laboratoire de Physiologie et

Physiopathologie en Conditions

Extrêmes, UMR MD2, IFR, Jean Roche,

Faculté de Médecine Nord, Aix-Marseille

Univ, Marseille; 2UFR de Biologie,

Université Joseph Fourier, Grenoble and

Laboratoire de Physiologie et

Physiopathologie en Conditions

Extrêmes, UMR MD2, IFR Jean Roche,

Faculté de Médecine Nord, Aix-Marseille

Univ, Marseille, France

\section{Abstract}

Molecular mechanisms underlining hypoxia-induced aged-hearing loss were studied. 3months C57BL/6 mice were subjected to four weeks of hypoxia $\left(10 \% 0_{2}\right)$, whereas, controls were kept under normoxic condition for up to six months. Auditory function was explored by CAP and Preyer's reflex measurements and correlated with histological analysis of the cochlea. The presence of oxidative damage, HIF-1 responsive target genes regulation involved in cell death, inflammation and neovascularization were assessed by immunofluorescence analysis. Hypoxia was associated to severe hearing loss at $4-8$ and $16 \mathrm{KHz}$ and degeneration of the cochlea, with significant cell loss (30\%) in the spiral ganglion, the lateral wall, and the hair cells with a basal-apical alteration gradient. This was correlated with ROS formation and HIF-1 $\alpha$ overexpression. Cochlear degeneration was due to apoptosis via activated caspase-3, P53, Bax and Bcl-2 protein differential expression in spiral ganglion, modiolus and spiral ligament. On the other hand, Hsp70, NF-kB transcription factor pathway and inflammatory mediators (caspase-1 and TNF- $\alpha$ ) were induced in the stria vascularis. Furthermore, a phenomenon of neovascularization was observed with significant thickening of stria vascularis and increased expression of VEGF. In total, we demonstrated that the tandem-HIF-ROS is responsible for the caspase- 3 and Bax-mediated apoptosis via P53 protein accumulation in the cochlear neurons, while inflammatory response mediated by Hsp70 stress protein and NF-kB transcription factor generating a neovascularization phenomenon occurred in stria vascularis.

\section{Introduction}

Normal functioning of the cochlea is highly dependant upon oxygen supply, as previously reported.1,2 In fact, it has been shown that hypoxia can cause reversible hearing loss, ${ }^{3}$ as well as damages in brain development ${ }^{4}$ and muscle degeneration. 5

There may be unique physiological signal associated with transitions between normoxia and hypoxia statuses, such as the formation of reactive oxygen species (ROS). Others as well as ourselves have demonstrated that ROS play a key role in mechanisms for induction of cochlear damage under various pathological conditions. ${ }^{6,7}$ Indeed, studies have shown that the reduction in oxygen availability to act as an electron sink that is normally associated with hypoxia may result in the accumulation of reducing equivalents in the mitochondrial electron transport chain, which can lead to augmented production of ROS. ${ }^{8}$ In previous reports, we have demonstrated that cell hypoxia by itself, can result in the formation of increased ROS level, resulting in the induction of apoptosis.7,9 Therefore, the regulation of ROS levels is important to prevent cochlear degeneration. In recent years, a greater appreciation has emerged of the role of ROS as signaling molecules in gene regulation. 10

Candidate target genes of transcription factors such as hypoxia inducible factor (HIF-1) ${ }^{10}$ is capable of transactivating many oxygen radical sensitive genes involved in different pathways promoting either inflammation (tumor necrosis factor TNF- $\alpha$, NF-kB, caspase-1), cell death (P53, the Bcl-2 family genes, caspase-3) or stress protein (HSP70). ${ }^{10}$ In addition, a relationship between aging, a vascular network and VEGF expression change was identified.11 VEGF, an endothelial cell-specific mitogen, is important for endothelial and metabolic functions, and especially vascular metabolism functions (vessel formation, relaxation, permeability, etc.), inflammation and apoptosis. The aim of the present study was to understand the mechanisms responsible for cochlear degeneration caused by the lack of oxygen directly affecting cochlear function. We used inbred C57BL/6 mouse strain that we exposed to continuous hypoxia (4 weeks). The main finding of this study is that hearing loss observed under hypoxia is associated to strong alteration of the spiral ganglion and the stria vascularis structures due to increasing formation of oxygen radicals in the cochlea. Subsequent disturbances of cellular signaling cascades implicating HIF-1 $\alpha$, Hsp70, VEGF, p53, Bcl-2 family proteins may lead ultimately to apoptosis (detected by activated caspase-3) with or without inflammation (presence of TNF- $\alpha$ and NF-kB).
Correspondence: Catherine Riva, Laboratoire de Physiologie et Physiopathologie en Conditions Extrêmes, UMR MD2 (P2COE), IFR Jean Roche, Faculté de Médecine Nord, Aix-Marseille Univ, Bd Pierre Dramard, 13916 Marseille cedex 20, France. Tel. +33.490162933 .

E-mail: catherine.riva-lavieille@univ-avignon.fr

Key words: cochlear neurons, hearing loss, apoptosis, hypoxia, VEGF, ROS, HIF-1 $\alpha$.

Acknowledgment: the authors would like to thank Michel Lucciano, Nathalie Kipson and Nabil Adjriou for their technical assistance and Christine Criado for her help in manuscript preparation. This work was supported by a grant from the University of Méditerranée Marseille France, and the Lion's Club, Bouches du Rhône Foundation, France.

Received for publication: 7 October 2010

Revision received: 6 October 2011.

Accepted for publication: 5 December 2011.

This work is licensed under a Creative Commons Attribution NonCommercial 3.0 License (CC BYNC 3.0).

(C) Copyright E. Donadieu and C. Riva, 2012

Licensee PAGEPress, Italy

Ageing Research 2012; 4:e1

doi:10.4081/ar.2012.e1

\section{Material and Methods}

The animal experiments were performed in strict accordance with the European Commission guidelines (86/609/EEC) and the National Animal Care guidelines. The local Veterinary Ethics Committee (Jean Roche Institute) approved the study.

\section{Animals}

Experiments were performed on $\mathrm{C} 57 \mathrm{BL} / 6$ male inbred mice aged three months $(n=31)$ and six months $(n=7)$ old (Charles River Laboratories, France) weighing 15-40 g. They were housed in living quarters with controlled temperature $\left(21 \pm 1^{\circ} \mathrm{C}\right)$, light (12-h light/12-h dark cycle, with the light switched on at 7 a.m.), ambient noise level in order to avoid stress in animals with genetic background,12 and with free access to food and water.

\section{Exposure to low oxygen environ- ment}

A group of 7 C57BL/6 3-month old mice (weighing 15-20g) was maintained for four weeks under normoxic conditions $\left(\mathrm{FiO}_{2}=\right.$ 21\%). A second group of $17 \mathrm{C} 57 \mathrm{BL} / 63$-month old mice was maintained four weeks under hypoxic conditions and breathed an inspired oxygen fraction $\left(\mathrm{FiO}_{2}\right)$ of $10 \%$ without $\mathrm{CO}_{2}$ 
accumulation as we described previously. 13 After the 4-week period, mice from the two groups were removed from the chamber and were immediately processed for blood sampling ( $n=7$ normoxic mice and $n=7$ hypoxic mice) for End-Tidal $\mathrm{O}_{2}$. Furthermore, animals (controls and hypoxic) were tested for $\mathrm{O}_{2}$ and $\mathrm{CO}_{2}$ fractions measurement as described previously by Arbogast et al. ${ }^{14}$

\section{CAP and Preyer's reflex (Pina twitch) audiometry}

The electrophysiological system used for CAP measurement in this study was similar to that described previously.15,16 Briefly, electrodes were implanted under stereotactic guidance near the auditory nerves of C57BL/6 mice, according to methods described by Giraudet and colleagues. ${ }^{17}$ After verification of an evoked potential in response to a broadband click, the electrode was fixed to the skull hole with acrylic cement (Dentalon Plus, Heraeus Kulzer, Weherheim, Germany). Animals were placed in a double-walled cabin (Amplifon, Milan, Italy), and cochlear sensitivity was assessed by measuring the threshold of the auditory nerve CAP at frequencies of $0.5,2,4$, 8,16 , and $32 \mathrm{kHz}$ with tone pips (rise and fall $2 \mathrm{~ms}$; rate $30 \mathrm{pips} / \mathrm{s}$ ) delivered by means of an earphone maintained on the animal's head by the fixed connector, at a distance of $0.5 \mathrm{~cm}$ from the ipsilateral ear pinna. SPLs were measured in decibels with a condenser microphone (model n. 4191, Bruel and Kjaer, Naerum, Denmark) placed at the external auditory meatus; for each frequency, we recorded 200 auditory responses to obtain an average SPL for comparison. Acoustic levels were calibrated and expressed in $\mathrm{dB}$ of sound pressure level (dB SPL).

In addition, the evaluation of Preyer's reflex (pinna twitch) was used by an adapted method of the Pinna reflex test described previously. 18,19 Briefly, this test was performed using a click box that produces standardized, calibrated sounds of 2, 4, 8 and $16 \mathrm{KHz}$. An earphone producing the sound was placed at the same distance above each animal's head by fixed connector, at a distance of $0.5 \mathrm{~cm}$ from the ipsilateral ear pinna. This Preyer's reflex test was performed in an interval time of a maximum 2 $\mathrm{h}$ after removing the animal from the hypoxic chamber. The reflex was considered positive when a rapid movement of the whole body of the animal was clearly noticed as detailed previously. 19

For each CAP and Preyer's reflex recording, the animals were placed in a double-walled cabin (Amplifon, Milan, Italy).

\section{Cochlea preparation}

All animals were sacrificed and both cochleae from every animal were dissected and perfused with a fixative containing 4\% paraformaldehyde in phosphate-buffered saline $(\mathrm{pH} 7.4)$ according to the technique described previously. ${ }^{2}$

\section{Histological and immunohisto- chemical investigations}

Serial $10 \mu \mathrm{m}$ thick sections of cochleae were mounted on gelatin-coated slides. They were then fixed for 10 min with 4\% paraformaldehyde and allowed to air dry at room temperature and stored at $4^{\circ} \mathrm{C}$. For histological examination, cochlea sections were stained with hematoxylin/eosin. Immunohistochemical analysis was performed as described previously. ${ }^{7}$ The primary antibodies used were mouse anti-active ${ }^{\circledR}$-caspase 3 monoclonal antibody (Promega ${ }^{\circledR}$, France), mouse anti-Bax (B9): sc7480 monoclonal antibody (Santa Cruz Biotechnology, CA, USA), mouse anti-VEGF (C1): sc-7269 monoclonal antibody (Santa Cruz Biotechnology, CA, USA), mouse anti-human Bcl-2 oncoprotein (M887) monoclonal antibody (DAK0), rabbit anti-HIF-1 $\alpha$ (H206): sc-10790 polyclonal antibody (Santa Cruz Biotechnology, CA, USA), goat anti-p53 (C19): sc-1311 polyclonal antibody (Santa Cruz Biotechnology, CA, USA), goat anti-TNF- $\alpha$ (N19): sc-1350 polyclonal antibody (Santa Cruz Biotechnology, CA, USA), rabbit anti-caspase-1 (A-19): sc-622 polyclonal antibody (Santa Cruz biotechnology, CA, USA), goat anti-NFKB p50 (C-19): sc-1190 polyclonal antibody (Santa Cruz Biotechnology, CA, USA) and rabbit anti-HSP-70 (AB3470) polyclonal antibody (Chemicon ${ }^{\circledR}$ Int., CA, USA), diluted in PBS-BSA 5\%. Secondary antibodies (Fluorescein (FITC)-conjugated AffiniPure $\mathrm{F}(\mathrm{ab})_{2}$ Fragment Goat anti-mouse, Donkey anti-mouse or Donkey anti-rabbit IgG and Rhodamine (TRITC)-conjugated AffiniPure $\mathrm{F}(\mathrm{ab})_{2}$ Fragment Donkey antimouse and Donkey anti-rabbit IgG were obtained from Jackson Immunoresearch (West Grove, PA, USA). To detect oxidized nucleosides, the 8-oxoG monoclonal antibody (Chemicon, Int., CA, USA) was used. Slides were then dehydrated, mounted in Mowiol (Calbiochem ${ }^{\circledR}$ ) containing DAPI (Calbiochem ${ }^{\circledR}$ ) for nuclei blue counter color and stored at $4^{\circ} \mathrm{C}$. Replacing the primary antibody with IgG1 or rabbit immunoglobulin fraction of serum controlled the specificity of the immunological reaction. Furthermore, cochleae vascularization studies were performed using a blood vessel staining kit (Peroxidase $\quad$ System, $\quad$ Chemicon ${ }^{\circledR}$ International). This kit utilizes the two most widely accepted cell markers for the study of angiogenesis and neovascularization: von Willebrand factor (Factor VIII Related Antigen) and CD31 (PECAM-1) antibodies. Chemicon's von Willebrand factor antibody reacts specifically with the endothelial cells of blood vessels and is a useful marker for the identification of endothelial lineage. 20

Signals were examined under Leica-DMR microscope (Leica Microsystems AG, Wetzlar, Germany) equipped for epifluorescence with $40 \mathrm{x}$ and $100 \mathrm{x}$ oil-immersion lenses. Images were acquired by using a cool CDD photometrics coolsnap camera (Roper scientific, Tucson, AZ, USA) and the image files were processed using CS2- Photoshop software (Adobe, San Jose, CA, USA). Parallel sections had been stained with hematoxylin/eosin or labeled with the different primary antibodies. Protein immuno-labeling was classified according to fluorescent signal abundance: absent, moderate and strong. The average evaluation of two fields, seen using a $10 \mathrm{x}$ objective lens, from each animal was evaluated. The area measurements of the SV such as length and thickness were obtained and calculated using Image J software (http://rsb.info. nih.gov/ij/, 1997-2006).

\section{Statistics}

The data are expressed as mean \pm SD. Statistical evaluation was performed by the non-paired Student's t-test to compare CAP thresholds and Preyer's reflex values at the different ages and before/after hypoxia exposition. Statistically significance was set at a level of $\mathrm{P}<0.05$. The number of histological observations is equal to the number of cochleae tested $(n=76)$. Student's t-test was also used to compare data regarding length and thickness of the stria vascularis obtained with Image J software. The difference was considered significant at $\mathrm{P}<0.05$.

\section{Results}

\section{CAP and Preyer's reflex (Pina twitch) audiograms}

Mean CAP threshold and Preyer's reflex values measured on normoxic C57BL/6 3-month old mice ( $n=7)$, normoxic C57BL6 6-month old mice $(n=7)$, and 4-month old hypoxic C57BL6 mice $(n=10)$ are presented in Table 1 . The results demonstrate that normoxic C57BL/6 3month old mice had a minimal CAP threshold of $48 \pm 5 \mathrm{~dB}$ observed at $16 \mathrm{KHz}$. No significant hearing loss between 3 -month and 6 -month old mice was observed in the normoxic group. However, after maintenance for four weeks in hypoxic conditions, we had a significant increase in thresholds at 4,8 and $16 \mathrm{KHz}$, especially a $20 \mathrm{~dB}$ hearing loss at 8 and $16 \mathrm{KHz}$ in comparison to C57BL/6 3-month old control mice. In addition, the hearing thresholds observed after four weeks of hypoxia were significantly higher to the CAP threshold measured in 6-month control mice, and turned to be 
equivalent of CAP thresholds of C57BL/6 14month old mice, as reported by Johnson et al. ${ }^{21}$

\section{Morphological analysis of the cochlea}

After examination of the sections of hypoxic C57BL/6 mouse cochleae, significant histological changes were observed, in comparison to 3 month old mice (Figure 1A). In both apical and basal turns of the cochlea, (Figure 1B and 2C), approximatively $30 \%$ of cell losses in the spiral ganglion (SG) were detected. Furthermore, losses and disorganization were observed in the organ of Corti (OC), the inner hair cells (ihc) and the outer hair cells (ohc). In the spiral ligament (SL), a basal-apical alteration gradient could be noted. In the apical turn, we observed fibrocytes types I and III total extinction and fibrocytes type II partial extinction (50\%) (Figure 1B). In contrast in the basal turn, only little fibrocytes II and III cellular losses were observed (Figure 1C). Furthermore, different morphological alterations of the stria vascularis (SV) between the basal and apical turns were quantified. Firstly, the length of SV was significantly longer in the basal turn in comparison to the apical turn whatever the mice groups, with 33,44 and $38 \%$ in length for 3-month, hypoxic and 6-month old mice cochlea, respectively. In addition, the SV displayed a phenomenon of shortening, after hypoxic treatment as well as in 6-month old mice with a decreased size of about $17 \%$ and $15 \%$ for hypoxic and 6-month old mice in the apical turn respectively in comparison to 3 -month old mice $(\mathrm{P}<0.01)$. The shortening of the SV region was not as pronounced in the basal turn of the cochlea in both situation in comparison to 3-month mice cochlea (Figure 2A). We also evaluated the thickness of the SV. We noted a thicker SV layer in basal turn of 3and 6-month mice cochlea than in apical turns (Figure 2B). After four weeks hypoxia, a significant thickening of the SV with more than $28 \%$ and $64 \%$ in depth in the basal turn and apical turn, respectively, was observed $(\mathrm{P}<0.01)$ (Figure 2B). In comparison, this phenomenon is not as intense in the cochlea of old mice (6month old) with only a thickening of the SV of $3 \%$ and $15 \%$ in size in the basal and apical turn, respectively (ns).

\section{Hypoxia triggers HIF-1 $\alpha$ expression}

To detect hypoxia in C57BL/6 mice, HIF-1 $\alpha$ protein expression was assessed by immunohistochemistry in the cochleae from control 3month $(\mathrm{n}=14)$, 4-month $(\mathrm{n}=14), 6$-month $(\mathrm{n}=14)$ and 4-month hypoxic $(\mathrm{n}=14)$ C57BL6 mice. A significant increased expression of HIF-1 $\alpha$ protein was noted in the cochleae of hypoxia-conditioned animals while no expression was observed in 3- and 6-month old control mice. These data are summarized in Table 2. The HIF-1 $\alpha$ immunolocalization in hypoxia-
Table 1. Comparison of CAP audiogram and Preyer's reflex in C57BL/6 mice.

\begin{tabular}{lccc} 
& \multicolumn{2}{c}{$\begin{array}{c}\text { CAP threshold } \\
(\mathrm{dB}) / \mathrm{SD}\end{array}$} & $\begin{array}{c}\text { Preyer's reflex } \\
(\mathrm{d}) / \mathrm{SD}\end{array}$ \\
Frequency $(\mathrm{Hz})$ & $\begin{array}{c}3-\text { month } \\
(\mathrm{n}=7)\end{array}$ & $\begin{array}{c}6 \text {-month } \\
(\mathrm{n}=7)\end{array}$ & $\begin{array}{c}\text { 4-month hypoxic } \\
(\mathrm{n}=10)\end{array}$ \\
500 & $103 / 0$ & $103 / 0$ & $\mathrm{~nm}$ \\
2000 & $95 / 0$ & $99 / 5$ & $100 / 5$ \\
\hline 4000 & $76 / 3$ & $80 / 3$ & $95 / 5$ \\
8000 & $55 / 3$ & $60 / 2$ & $75 / 3$ \\
16000 & $48 / 5$ & $60 / 3$ & $70 / 3$ \\
32000 & $92 / 0$ & $92 / 2$ & $\mathrm{~nm}$ \\
\hline
\end{tabular}

Nm, not monitored; SD, standard deviation.

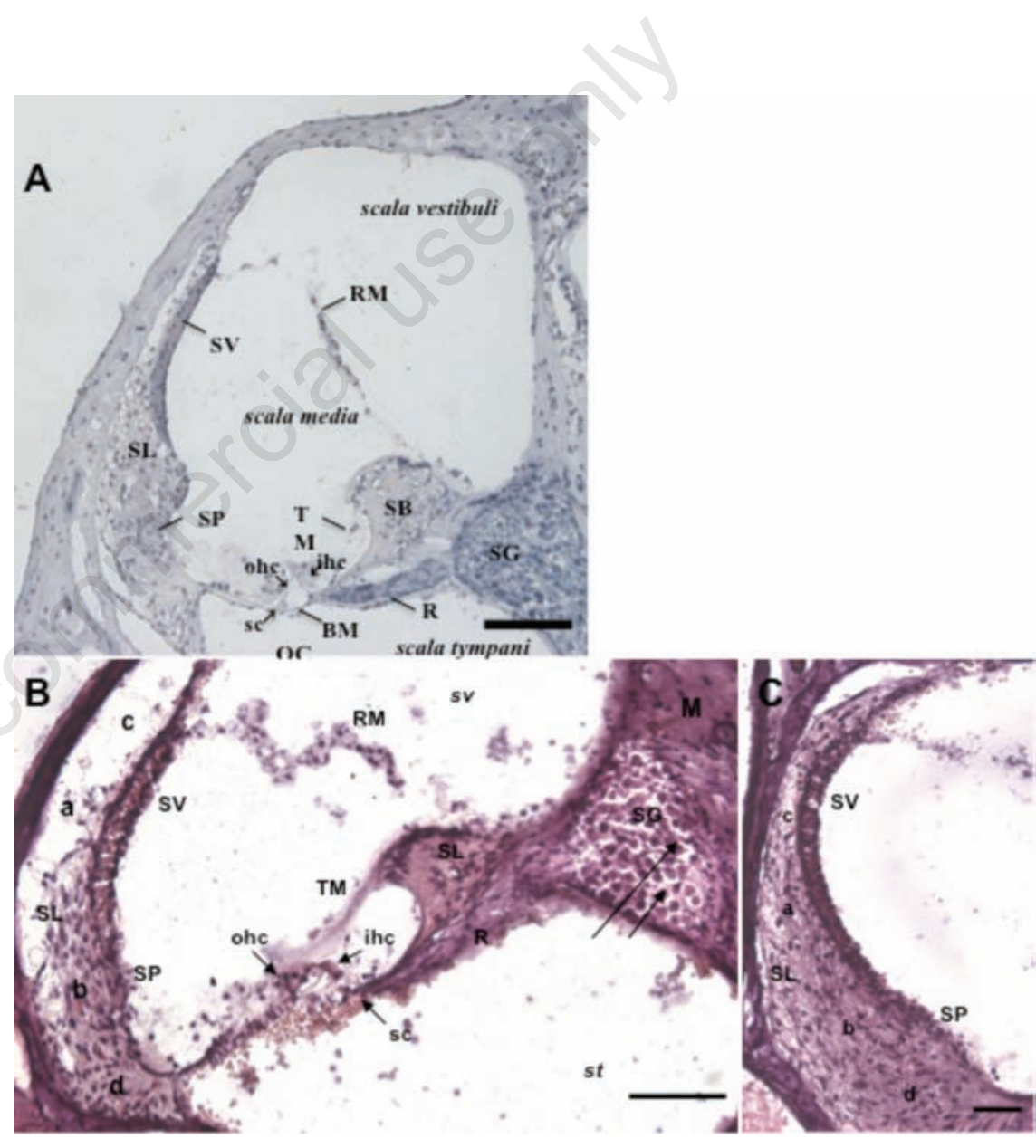

Figure 1. A) Light microscopy of a cross-section of hematoxylin/eosin stained C57BL/6 mouse cochlea showing the organ of corti (OC) and the lateral wall structures. The Reissner's membrane (RM) separating the scala vestibuli $(s v)$ and the scala media $(\mathrm{sm})$ could be observed as well as the OC delimitating the scala tympani (st). The spiral ganglion (SG), spiral limbus (SB), tectorial membrane (TM), basilar membrane (BM) and Rosenthal's canal $(\mathrm{R})$ are respectively noted. The $\mathrm{OC}$ was composed of the inner hair cells (ihc), the outer hair cells (ohc) and the supporting cells (sc). The lateral wall is composed of the stria vascularis (SV), the spiral prominence (SP) and the spiral ligament (SL). Scale bar: $50 \mu \mathrm{m}$. B) Cross-section of hematoxylin/eosin stained of a 4-month hypoxic C57BL/6 mouse apical turn cochlea. The SL was composed of types I (a), II (b), III (c) and IV (d) fibrocytes. Scale bar: $50 \mu \mathrm{m}$. C) Cross-section of haematoxylin/eosin stained of a 4-month hypoxic C57BL/6 basal turn mouse cochlea. Scale bar: $25 \mu \mathrm{m}$. 
conditioned animal cochleae, as technically reported before, ${ }^{2}$ showed differences in its regional distribution. It appeared to be highly expressed in the SG, the SV, the modiolus and the hair cells (Figure 3A). No significant expression was detected in the SL. No significant differences of HIF-1 $\alpha$ protein expression were found between upper and lower turns of the cochlea (data not shown).

\section{Hypoxia induced ROS generation}

Intracellular ROS were detected intensively within the SG, the modiolus and the SV of the cochleae of mice exposed to chronic hypoxia. The staining was diffused in the cytoplasm of the SG cells and present homogeneously in the SV (Figure 3B). On the contrary, no ROS signal was seen in control at 3-, 4- and 6-month old mice cochleae. Results are summarized in Table 2.

\section{Hypoxia triggers apoptosis in the cochlea}

After hypoxia, activated caspase-3 was intensively expressed in the cytoplasm of the $\mathrm{SG}$ and to a much lower extent in the SV of the cochleae of mice (Figure 3D). While no sign of activated caspase- 3 was detected at 3 -month old mice cochleae, weak expression of activated caspase-3 was detected in hair cells of 6 month old mice cochleae. Results are summarized in Table 2. The slides were counterstained with DAPI in order to assess the different aspects of cell nuclei and confirm that apoptosis occurred in neurons of the spiral ganglion (Figure 3E and $\mathrm{F}$ ).

The triple staining with HIF-1 $\alpha, 8-0 \times 0 \mathrm{G}$, and activated caspase-3 revealed that apoptosis and ROS formation were mainly present in cellular zones highly affected and sensitive to hypoxia, such as the spiral ganglion.

As previously reported,7,16 we hypothesized that proteins of the mitochondrial cell death pathway could interfere in the hypoxia-induced apoptosis in the cochlea. P53, Bax and Bcl-2 proteins expression was studied in our hypoxic C57BL/6 mouse model.

\section{Increased P53 protein expression}

In the C57BL/6 mice cochleae exposed to hypoxia, positive $\mathrm{P} 53$ reactivity was seen in the SG, in the soma of the neurons with more than $50 \%$ of positive cells and a cytoplasmic localization of the protein, and in the SV regions, while in control 3-, 4- and 6-month C57BL/6 mice cochleae, P53 immunohistochemical staining was either weak or absent in these regions. Negative and positive controls for P53 were performed according to our previous report. 22

Increased in $\mathrm{Bax}$ and $\mathrm{Bcl}-2$ proteins expression

Bax and Bcl-2 protein expression was assessed in the C57BL/6 normal and hypoxic mice cochleae. Apoptotic spiral ganglion cells,
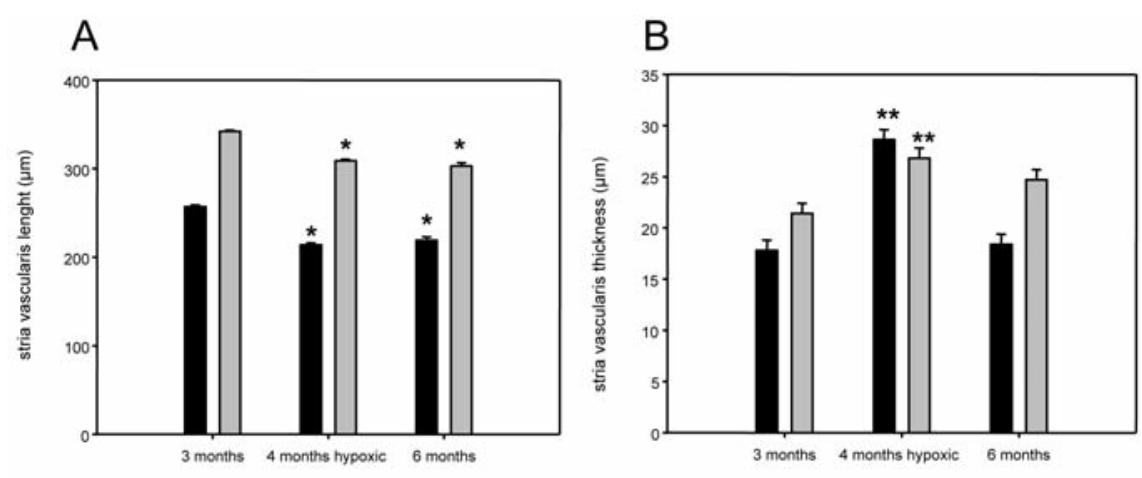

Figure 2. A) Distribution of stria vascularis basal (black) and apical (gray) turns thickness $(\mu \mathrm{m})$ in 3-month old control $(n=5)$, 4-month hypoxic $(n=5)$, and 6 -month old C57BL/6 mice $(n=5)$. The difference between the 4-month hypoxic C57BL/6 mice, and the 6month old C57BL/6 mice group in comparison to 3-month old control mice was significant for basal and apical turns $\left(^{*}\right)(P<\mathbf{0 . 0 5})$. Errors bars represent standard deviations. $\left.\mathrm{B}\right)$ Distribution of stria vascularis basal (black) and apical (gray) turns lenghts $(\mu \mathrm{m})$ in 3month control $(n=5)$, 4-month hypoxic $(n=5)$ and 6 -month control C57BL/6 mice $(n=5)$. The difference between 4-month hypoxic C57BL/6 mice and 3-month old mice control group was significant for the basal and apical turns $(* *)(P<0.01)$. Error bars represent standard deviations.

Table 2. Summary of stress-protein immunohistochemical detection and presence of ROS.

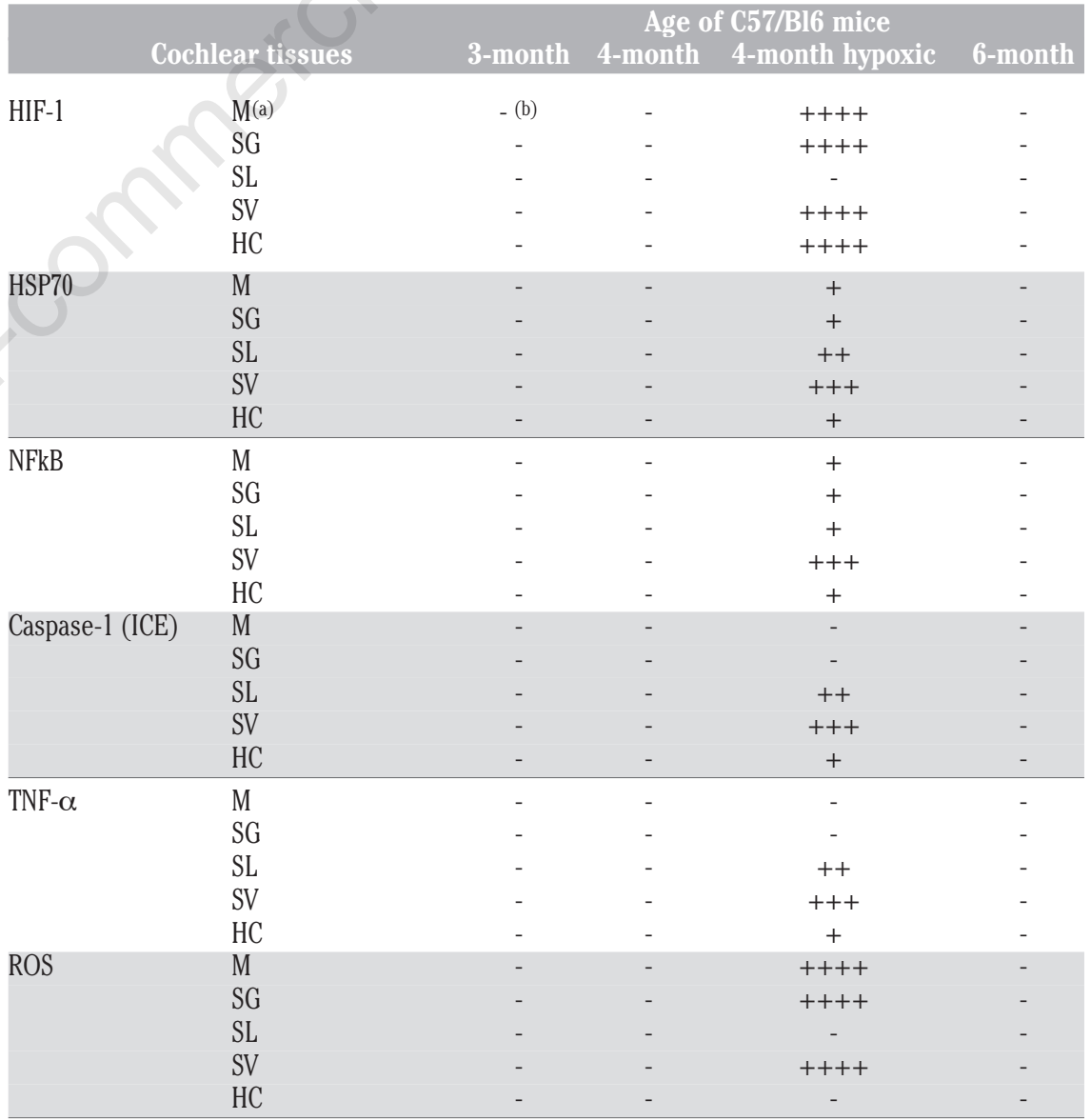

aM, modiolus; SG, spiral ganglion; SL, spiral ligament; SV, stria vascularis; HC, hair cells. bscore -, negative staining; +, 1-20\% of positive cells; ,$++ 21-59 \%$ of positive cells;,$+++ 60-80 \%$ of positive cells;,$++++>80 \%$ of positive cells. 
as shown above in $\mathrm{C} 57 \mathrm{BL} / 6$ mice cochleae exposed to hypoxia, were stained positive with Bax antibody. Bax and Bcl-2 immunohistochemical staining appeared in the spiral ganglion cells cellular bodies. After four weeks exposure to hypoxia, about $80 \%$ of cells in the spiral ganglion cells were stained positive for activated caspase-3. Bax and Bcl-2 has not been observed in 3-, 4- and 6-month control C57BL/6 mice cochleae and weak expression was also noted in hair cells of 6 -month old mice. In addition, Bax and Bcl-2 immunohistochemical staining appeared in the remaining SL fibrocytes types II and IV of the 4-month C57BL/6 mice cochleae exposed to hypoxia. P53, Bax and Bcl-2 expressions are summarized in Table 3.

\section{Hypoxia induces Hsp-70 expression}

No expression was noted on cochlea of 3-, 4and 6-month old control mice. When the mice were subjected to hypoxia, there was a significant increase in expression of HSP70, but in distinct regions such as SV (Figure 3G), to a lesser extent in the SL and to a very low level in neural cells of the SG and modiolus as well as in hair cells (Table 2). The triple staining with activated caspase-3, 8-oxoG, and Hsp70 demonstrated that $\mathrm{Hsp} 70$ was also expressed in the zone affected by ROS generation but not where apoptosis was detected. We conclude that hypoxia triggered HIF-1 $\alpha$, Hsp70 and ROS formation which may induce either apoptosis in a selected neural zone of mice cochlea, such as the SG and in the SL or protection in the SV.

\section{Hypoxia stress produces inflamma- tion in the cochlea}

To test if hypoxia could cause inflammation, we measured NFK-B induction, caspase- 1 and TNF- $\alpha$ expression in the cochlea. 23 There was a significant increase in the NFK-B level in the nuclei of cells of the SV and to a lower extent in the SL of hypoxic cochlea (Figure 3H). In the other cochlear regions, no significant signal for NFK-B was detected either in control or hypoxic cochleae. While no expression of caspase- 1 or TNF- $\alpha$ could be detected in cochlea of 3-, 4- and 6-month old control C57BL/6 mice, remarkably these proteins were present in the cochlea of hypoxic C57BL/6 mice around the SV and the SL, interestingly in regions where hypoxia was detected. No expression of TNF- $\alpha$ was detected in the SG neurons of cochlea either in control C57BL/6 mice or in mice exposed to hypoxia. The results are summarized in Table 2.

\section{Neovascularisation detection}

To understand the SV morphological alterations previously observed, we studied vascularization with a blood vessel staining kit, as described in Materials and Methods. This test contains anti-von Willebrand and anti-CD31 antibodies specific for the detection of vascular endothelial cells. We compared the 4-month old hypoxic C57BL/6 mouse model to the 3-, 4and 6-month old control C57BL/6 mice. Figure 4 demonstrated a strong staining of the SV layer in hypoxic cochleae in comparison to 3and 6-month old mice (Figure 4A and $\mathrm{C}$ ). This acute vascularization in hypoxic cochlea can be confirmed by the changes in SV length and thickness observed and described above (Figure 2A and B).
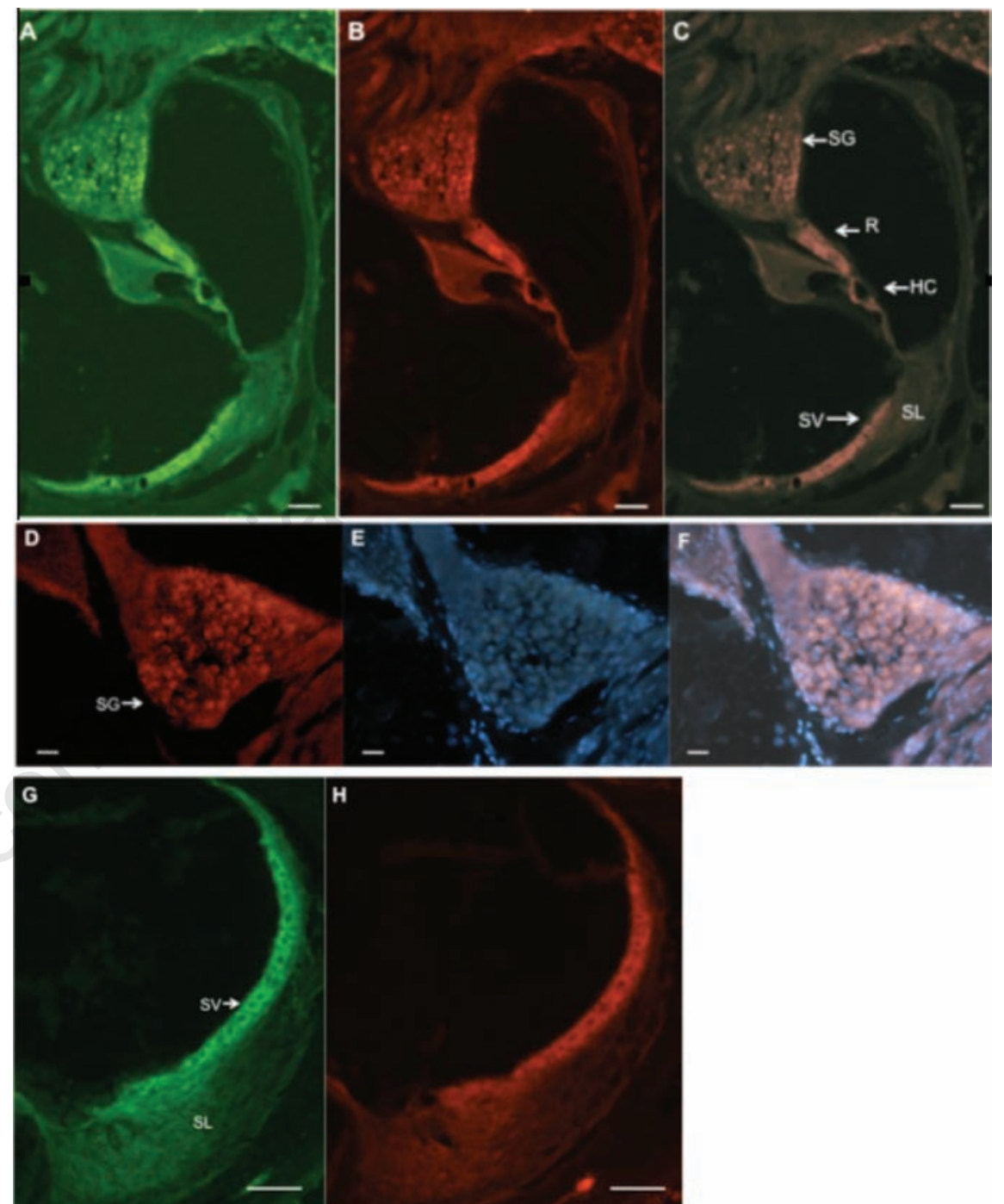

Figure 3. Photomicrographs of immunofluorescent images taken from cochleae of 4-week hypoxia exposed $\mathrm{C} 57 \mathrm{BL} / 6$ mice. Five micrometers sections of the cochlea were subjected to immunohistochemical analysis with specific antibodies as described in Material and Methods. (A) Expression of HIF-1 $\alpha$ protein, (B) detection of oxygen radicals by 8-oxoG staining and (C) merged. There was no staining in spiral ligament (SL). In contrast, it is expressed essentially in the spiral ganglion (SG), the stria vascularis (SV), the hair cells (HC) and in the Rosenthal canal (R). The presence of ROS was clearly co-localized with over-expressed HIF-1 $\alpha$ protein. Original magnification 100X (scale bars a, b, c: $50 \mu \mathrm{m}$ ). (D). Caspase-3 positive cells were detected in spiral ganglion (SG). (E) Counterstaining of the spiral ganglion neurons with DAPI, (F) merge, note that nuclei of neurons can be observed. Original magnification 400X (scale bars d, e and f: $10 \mu \mathrm{m}$ ). (G) HSP-70 and H/ NFKB were significantly expressed in the stria vascularis (SV) but not in spiral ligament (SL). Original magnification 400X. (scale bars g and h: $50 \mu \mathrm{m})$. 
which confirms the intracellular localization VEGF (Figure 3E and F). On the other hand, no significant VEGF staining was detected in 3-, 4- and 6-month control C57BL/6 mice cochleae (data not shown). Finally, we demonstrated that the regional change of HIF-1 $\alpha$ expression was parallel to the VEGF expression, confirming that HIF-1 $\alpha$ regulates the VEGF expression in the cochlea.

\section{Discussion}

Our present study focused on the role of hypoxia on cochlear degeneration and its subsequent disturbances of cellular signaling cascades that lead to the aged-related hearing loss or presbyacusis. We used a C57BL/6 mouse model, which possessed the adult hearing loss gene $(A h l)$ characterized as recessive and mapped to chromosome 10, presumed to cause progressive hearing loss. In fact, we and others have reported that, at six months of age, these animals show a significant hearing loss at high frequencies $(4,8 \text { and } 16 \mathrm{kHz})^{12,24}$ which reaches lower frequencies after 12 months. ${ }^{21}$ This functional deficit is strongly associated with degenerative histopathological changes. In fact, in the C57BL/6 mice, aged three and four months, no significant alteration of the cochlear histology was observed. The major morphological change appeared at six months and was the degeneration of the outer hair cells of the organ of Corti. The extended alteration to the inner hair cells and the spiral ganglion as reported by Mikaelian et al. ${ }^{25}$ occurred later at ten months. In addition, as Ohlemiller showed, ${ }^{26}$ on 6 -month old $\mathrm{C} 57 \mathrm{BL} / 6$ mice, the cochlea basal turn is affected and extension to the apical turn occurred after 12 months. These alterations involved the lateral wall, and in particular the SV.

Physiological and metabolic consequences of hypoxia are largely recognized in experimental models. ${ }^{27}$ In our study, a group of C57BL/6 mice was maintained under hypoxic conditions $\left(\mathrm{FiO}_{2} 10 \%\right.$, corresponding to oxygen pressure found at an altitude of about 5,500 m), ${ }^{28}$ for a period of four weeks; so called chronic hypoxia. Therefore, during this chronic hypoxia period, where arterial $\mathrm{PO}_{2}$ and venous $\mathrm{PO}_{2}$ levels were maintained at such a low level $(<30 \mathrm{mmHg}$ and $<22 \mathrm{mmHg}$, respectively; data not shown), tissues such as the cochlea would not receive sufficient oxygen, which could result in a deleterious effect on the hearing system. In fact, our results clearly demonstrated that four weeks of hypoxia resulted in significant hearing loss of more than $20 \mathrm{db}$ at frequencies of 4,8 and $16 \mathrm{kHz}$. These results are different from those of Chen ${ }^{29}$ who showed that $4 \mathrm{~h}$ hypoxia did not result in hearing loss, but hypoxia conditions such as acute for $4 \mathrm{~h}$ hypoxia are considerably different from chronic hypoxia for four weeks. ${ }^{28}$ The histological modifications observed in cochlea of four week hypoxic mice were quite different to those noted in 6-month old C57BL/6 mice, ${ }^{30}$ and were more related to those encountered in the rapidly ageing $\mathrm{cd} / 1$ mice model that we described previously, ${ }^{7}$ where SG and lateral wall cells were precociously affected. The cellular loss (30\%) quantified in the SG of four weeks hypoxic cochleae, are in agreement with White et al. ${ }^{30}$ and their concept of retrograde degeneration of the SG in very old (12-18 months) C57BL/6 mice, but contradictory to Stamataki et al.31 who did not observe any SG cells loss during

Table 3. Summary of apoptosis-protein immunohistochemical detection.

\begin{tabular}{|c|c|c|c|c|}
\hline & Cochlear tissues & 3-month & $\begin{array}{c}\text { Age of C57/BL6 mice } \\
4 \text {-month hypoxic }\end{array}$ & 6-month \\
\hline Caspase-3 & $\mathrm{M}^{(\mathrm{a})}$ & - (b) & ++++ & + \\
\hline & SG & - & ++++ & + \\
\hline & SL & - & - & - \\
\hline & SV & - & ++++ & - \\
\hline & $\mathrm{HC}$ & - & - & ++ \\
\hline $\mathrm{P} 53$ & M & + & ++++ & + \\
\hline & SG & + & ++++ & + \\
\hline & SL & - & - & - \\
\hline & SV & + & ++++ & + \\
\hline & $\mathrm{HC}$ & - & - & - \\
\hline Bax & M & + & ++++ & - \\
\hline & SG & + & ++++ & - \\
\hline & SL & - & ++++ & - \\
\hline & SV & & ++++ & - \\
\hline & $\mathrm{HC}$ & & + & ++ \\
\hline $\mathrm{Bcl}-2$ & M & - & ++++ & - \\
\hline & SG & - & ++++ & - \\
\hline & SL & - & ++++ & - \\
\hline & SV & - & ++++ & - \\
\hline & $\mathrm{HC}$ & - & + & ++ \\
\hline
\end{tabular}

aM, modiolus; SG, spiral ganglion; SL, spiral ligament; SV, stria vascularis; HC, hair cells. bscore -, negative staining; +, 1-20\% of positive cells; ,$++ 21-59 \%$ of positive cells;,$+++ 60-80 \%$ of positive cells;,$++++>80 \%$ of positive cells.
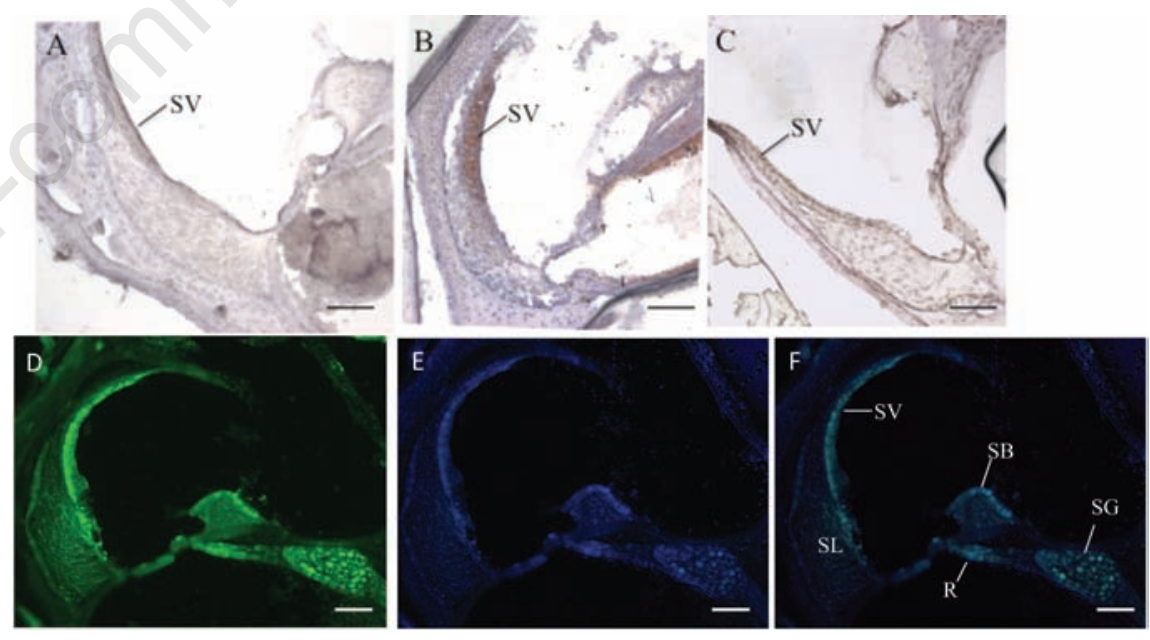

Figure 4. Cross-sections of C57BL/6 mouse cochlea stained with blood vessel staining kit. (A) Cochlea of 3-month control C57BL/6 mouse. No significant staining was visible in the interest structures. Scale bar: $50 \mu \mathrm{m}$. (B) Cochlea of 4-month hypoxic C57BL/6 mouse. It was exclusively stained in the basal and apical turns stria vascularis (SV) and in

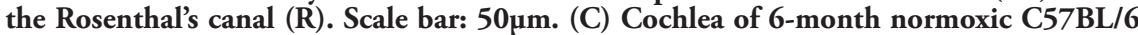

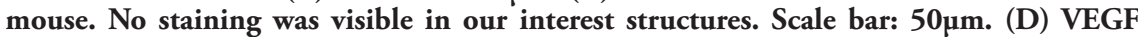
immunostaining of cochlea of hypoxic C57BL/6 mice using anti-VEGF antibody was intense in the stria vascularis (SV), while a weaker staining was observed in the spiral ganglion (SG), the spiral ligament (SL), the Rosenthal's canal (R) and the spiral lymbus (SB). (E) Cochlea section counterstained with DAPI. (F) Merged. Magnification 100X (Scale bars: $d$, e and $f: 50 \mu \mathrm{m})$. 
ageing but only a decrease in afferent terminals in older C57BL/6 mice (8-12 months). In addition, we have observed structural degenerations in the SL (fibrocytes types I, II and III) at the apical turn of the cochlea, whereas in the basal turn, the same structural alterations were observed except for type I fibrocytes, which were not affected. These SL modifications are in line with the results reported by Spicer and Schulte ${ }^{32}$ on aged gerbils. So, we can conclude in a basal-apical structural alterations gradient in the SL. Furthermore, SV important structural length and thickness modifications were observed. This is in agreement with previous reports. ${ }^{33,34}$ Effectively, the atrophy of the lateral wall and especially the SV is correlated with presbyacusis pathology. Reporting to its ion transport system properties, which degenerate with age,, 33 observations of SV atrophy seem logical.

An important transcription factor involved in the signaling and adaptation of hypoxia/ischemia is the hypoxia inducible factor (HIF). 35 Using a technique developed previously, ${ }^{2}$ we did not reveal any significant HIF-1 $\alpha$ expression in cochlea from mice aged three, four and six months. On the contrary, HIF-1 $\alpha$ was detected in cochleae of the 4-month hypoxic C57BL/6 mice with a differential region specific pattern of HIF activation that may indicate that HIF expression plays a role in inner ear homeostasis. The highest expression observed in $\mathrm{C} 57 \mathrm{BL} / 6$ mice exposed to hypoxia was found in the SG, the modiolus and the SV. In fact, the most hypoxia sensitive cells, such as the ganglion neurons and the Schwann cells ${ }^{36}$ are effectively localized in the region of the SG. These results are in line with findings of Stroka et al. ${ }^{37}$ and Gross et al. ${ }^{38}$ who showed that functionally different cochlea regions explanted in vitro have a specific HIF$1 \alpha$ expression. In our in vivo study, we demonstrated clearly that HIF-1 $\alpha$ protein, effectively expressed in specific areas, is the cogent demonstration of the presence of a hypoxic environment in the cochlea, as previously reported. ${ }^{2}$

It has been proposed that increased ROS generation would allow the hypoxic response 39 by inducing the post-translational stabilization of HIF-1 $\alpha$ during hypoxic incubation of cells and may further cause perturbation of the downstream transactivation of HIF-1 $\alpha$ target genes. We estimated the ROS formation in the cochlea in response to the chronic hypoxia condition. We found nuclear immunoreactivity for 8-0xoG corresponding to genomic oxidative DNA damage according to Donadieu et $a l .{ }^{2}$ specifically in the SG and the SV of cochlea of hypoxic C57BL/6 mice, while there was no labeling for 8-0xoG in cochleae of 3-, 4and 6 -month mice. The regional and temporal patterns of alteration due to oxidative damage and hypoxia were quite consistent.
Since heat shock proteins have been shown to have cytoprotective function, we addressed how Hsp70 contributed to protection against hypoxia injury. It has been previously reported that Hsp70 signaling is under the control of HIF-1 $\alpha$ expression in cells such as kidney. ${ }^{40}$ In our study, we showed the upregulation of Hsp70 in SV of the cochlea of hypoxic C57BL/6 mice, demonstrating that it may enhance the resistance of cells of SV to hypoxia-induced apoptosis, since lower activated-caspase-3 expression was noted in these zones. These findings provide a new insight into how cells overcome hypoxic stress and survive, and also disclose a new regulatory mechanism of $\mathrm{Hsp70}$ expression in auditory cells. However, the overall bad response to hypoxic stress in the cochlea may be partly due to the impaired Hsp70 induction of the different cells of the cochlea. 41

In fact, if rescuing pathways in cells after the hypoxic stress are deficient, the consequence is programmed cell death (apoptosis) by induction of the expression of different genes such as Bcl-2 family like Bax protein, 42 P53 and caspase-3 protein, as we reported previously. ${ }^{7}$ In our study, in 3- and 4-month old C57BL/6 mice, apoptosis phenomenon did not occur since activated caspase-3 was not detectable in any region of the cochlea. However, activated-caspase- 3 was detected in SG cells in 6-month old animals, while activated caspase- 3 was clearly detectable in SG cells and SL of cochleae of mice exposed to four weeks hypoxia. In addition, we found that Bcl2 and Bax proteins were clearly present in C57BL/6 hypoxic mice cochleae in the SG, in the SL fibrocytes II and IV, interestingly in the same regions affected by the hypoxic stress. Furthermore, the localization of caspase- 3 positive cells correlated with the sites of ROS formation observed in the hypoxic cochleae.

On the other hand, activated HIF-1 factor, transcriptionally up-regulates a set of target genes involved in angiogenesis. ${ }^{43}$ We evaluated vascular endothelial growth factor (VEGF) expression and secondly inflammatory response. In C57BL/6 mice exposed to four weeks hypoxia, the presence of VEGF was restrained to highly vascularized zones, the SV and in the nervous cells, SG and the modiolus. On the one hand, slight VEGF immunoreactivity was detected in the 3- and 4-month old C57BL/6 mice, and no significant VEGF expression was seen in 6-month old C57BL/6 mice, while a significant increase in staining was detected in the cochleae of four weeks hypoxic C57BL/6 mice. These findings are similar with previous studies ${ }^{11}$ that indicated that VEGF activity dramatically decreases with age, as we have demonstrated the decreased expression from three to six months. Furthermore, they indicated that hypoxia did induce VEGF expression and recruitment of vascular endothelial cells in selective zones, such as SV, compatible with neoangiogenesis phenomenon. In fact, in the C57BL/6 hypoxic mice group, the VEGF pattern expression was correlated to a neovascularization phenomenon, as previously reported by Picciotti et al. ${ }^{11}$ and Thomopoulos et al. ${ }^{44}$ who reported that vascular modifications of the inner ear are involved in age-related hearing loss. VEGF being crucial for endothelial function and metabolism, it may play an important role in the promotion of angiogenesis and the maintenance of an efficient tissue vascularization.

Hypoxia has also been reported to regulate the nuclear transcription factor (NF-kB) since this has been shown to mediate HIF-1 transcription. 45 In our study, NF-kB was highly expressed in the SV region of hypoxic cochleae. This overexpression was associated to the presence of cytokines interleukin-l (IL1) and tumor necrosis factor (TNF $\alpha$ ), which have been implicated in inflammatory responses. The increase in cytokine production during chronic hypoxia could be interpreted as a physiological response in the cochlea rather than a primary inflammatory mechanism, as reported previously by Moreau et al. 46

Altogether, the study of inflammatory mediator production and localization in the cochlea, showed a specific effect of HIF-1 mediated angiogenic factor (VEGF) during chronic hypoxia in the zone of SV compared to normoxic and aged control cochlea. This is in contrast to an early and non-specific activation of the NFK-B pathway with overproduction of IL-1 and TNF $\alpha$ in SV, as well as SL zones. All these results are consistent with selective pathway activation in the different regions of the cochlea with regard to their sensitivity to oxygen or ROS. The role of HIF-1 $\alpha$ in mediating both, pro-death, pro-survival or pro-inflammatory response is dependent, in this system, on the cell type it is induced in, as reported before by Vangeison et al. 47 However, much more information is needed to clarify the role of HIF in the hypoxia signaling pathway and its interaction with ROS, which in turn will enable us to design a therapeutic approach to degeneration of the cochlea based on a new antioxidant concept.

\section{References}

1. Cazals Y, Wu ZY, Horner K. Alterations of auditory nerve responses by hypoxia in normal and hydropic ears of awake guinea pigs. Hear Res 1994;77:177-82.

2. Donadieu E, Hamdi W, Deveze A, et al. Improved cryosections and specific immunohistochemical methods for detecting hypoxia in mouse and rat cochleae. Acta Histochem 2007;109:177-84. 
3. Attias J, Sohmer H, Gold S, et al. Noise and hypoxia induced temporary threshold shift in rats studied by ABR. Hear Res 1990;45:247-52.

4. Sheldon RA, Osredkar D, Lee CL, et al. HIF1 alpha-deficient mice have increased brain injury after neonatal hypoxia-ischemia. Dev Neurosci 2009;31:452-8.

5. Cerretelli P. Muscle energetics and ultrastructure in chronic hypoxia, Respiration 1992;59:24-9.

6. Ohlemiller KK, Dugan LL. Elevation of reactive oxygen species following ischemiareperfusion in mouse cochlea observed in vivo. Audiol Neurootol 1999;4:219-28.

7. Riva C, Donadieu E, Magnan J, Lavieille JP. Age-related hearing loss in CD/1 mice is associated to ROS formation and HIF target proteins up-regulation in the cochlea. Exp Gerontol 2007;42:327-36.

8. Poyton R0, Ball KA, Castello PR. Mitochondrial generation of free radicals and hypoxic signaling. Trends Endoc Metab 2009;20:332-40.

9. Riva C, Chauvin C, Pison C, Leverve X. Cellular physiology and molecular events in hypoxia-induced apoptosis. Anticancer Res 1998;18:4729-36.

10. Kietzmann T, Görlach A, Reactive oxygen species in the control of hypoxia-inducible factor-mediated gene expression. Semin Cell Dev Biol 2005;16:474-86.

11. Picciotti P, Torsello A, Wolf FI, et al. Agedependent modifications of expression level of VEGF and its receptors in the inner ear. Exp Gerontol 2004;39:1253-8.

12. Naff KA, Riva CM, Craig SL, Gray KN. Noise produced by vacuuming exceeds the hearing thresholds of C57BV/6 and CD1 mice. J Am Assoc Lab Anim Sci 2007;46:52-7.

13. Riva C, Chevrier C, Pasqual N, et al. Bcl2/Bax protein expression in heart, slowtwitch and fast-twitch muscles in young rats growing under chronic hypoxia. Mol Cell Biochem 2001;226:9-16.

14. Arbogast S, Darques JL, Jammes Y. Interactions between endogenous nitric oxide and hypoxemia in activation of group IV muscle afferents. Muscle Nerve 2002;96:194-200.

15. Cazals H., Huang ZW. Average spectrum of cochlea activity: a possible synchronized firing, its olivo-cochlear feedback and alterartions under anesthesia. Hear Res 1996;101:81-92.

16. Riva C, Longuet $M$, Lucciano $M$, et al. Implication de l'apoptose mitochondriale dans la dégénérescence neurale de la cochlée dans un modèle murin de presbyacousie. Rev Laryngol Otol Rhinol 2005;126:67-74.

17. Giraudet F, Horner KC, Cazals Y. Similar half-octave TTS protection of the cochlea by xylazine/ketamine or sympathectomy. Hear
Res 2002;174:239-48.

18. Cheatham MA, Pearce M, Richter CP, et al. Use of the pinna reflex as a test of hearing in mutant mice. Audiol Neurootol 2001;6:79-86.

19. Jero J, Coling DE, Lalwani AK. The use of Preyer's reflex in evaluation of hearing in mice , Acta Otolaryngol 2001;121:585-9.

20. Pusztaszeri MP, Seelentag W, Bosman FT. Immunohistochemical expression of endothelial markers CD31, CD34, von Willebrand factor, and Fli-1 in normal human tissues. J Histochem Cytochem 2006;54:385-95.

21. Johnson KR, Zheng QY, Erway LC. A major gene affecting age-related hearing loss is common to at least ten inbred strains of mice. Genomics 2000;70:171-80.

22. Cuisnier 0, Serduc R, Lavieille JP, et al. Chronic hypoxia against $\gamma$-irradiationinduced apoptosis by inducing bcl-2 up-regulation and inhibiting mitochondrial translocation and conformational change of bax protein. Int J Oncol 2003;23:1033-41.

23. Baker SJ, Reddy EP, Modulation of life and death by TNF receptor super family, Oncogene 1998;17:3261-70.

24. Li HS, Borg E. Age-related loss of auditory sensitivity in two mouse genotypes. Acta Otolaryngol 1991;111:827-34.

25. Mikaelian DO. Development and degeneration of hearing in the $\mathrm{C} 57 / \mathrm{b} 16$ mouse: relation of electrophysiologic responses from the round window and cochlear nucleus to cochlear anatomy and behavioral responses. Laryngoscope 1979;89:1-15.

26. Ohlemiller KK. Contributions of mouse models to understanding of age and noise hearing loss. Brain Res 2006;1091:89-102.

27. Connett RJ, Honig CR, Gayeski TE, Brooks GA. Defining hypoxia: a systems view of V02, glycolysis, energetics, and intracellular P02. J Appl Physiol 1990;68:833-42.

28. Peacock AJ. ABC of oxygen: Oxygen at high altitude. BMJ 1998;317:1063-6.

29. Chen GD. Effects of hypoxia on noiseinduced auditory impairment. Hear Res 2002;172:186-95.

30. White JA, Burgess BJ, Hall RD, Nadol JB. Pattern of degeneration of the spiral ganglion cell and its processes in the C57BL/6J mouse. Hear Res 2000;141:12-8.

31. Stamataki S, Francis HW, Lehar M, et al. Synaptic alterations at the inner ear hair cells precede spiral ganglion cell loss in aging C57BL/6J mice. Hear Res 2006;221:104-18.

32. Spicer SS, Schulte BA. Spiral ligament pathology in quiet-aged gerbils. Hear Res 2002;172:172-85.

33. Schulte BA, Schmiedt RA. Lateral wall Na,KATPase and endocochlear potentials decline with age in quiet-reared gerbils. Hear Res 2002;61:35-46.
34. Suzuki T, Nomoto Y, Nakagawa T, et al. Agedependent degeneration of the stria vascularis in human cochlea. Laryngoscope 2006;116:1846-50.

35. Semenza GL. Regulation of oxygen homeostasis by hypoxia-inducible factor 1 , Physiology (Bethesda) 2009;24:97-106.

36. Iida H, Schmeichel AM, Wang Y, et al. Schwann cell is a target in ischemia-reperfusion injury to peripheral nerve. Muscle and Nerve 2004;30:761-6.

37. Stroka DM, Burkhardt T, Desbaillets I, et al. HIF-1 is expressed in normoxic tissue and displays an organ-specific regulation under systemic hypoxia. FASEB J 2001;15:244553.

38. Gross J, Rheinländer C, Fuchs J, et al. Expression of hypoxia-inducible factor-1 in the cochlea of newborn rats. Hear Res 2003;183:73-83.

39. Chandel NS, Maltepe E, Goldwasser E, et al. Mitochondrial reactive oxygen species trigger hypoxia-induced transcription. Proc Natl Acad Sci USA 1998;95:1715-20.

40. Yeh CH, Hsu SP, Yang CC, et al. Hypoxic preconditioning reinforces HIF-1 $\alpha$ dependent Hsp70 signaling to reduce ischemic renal failure-induced renal tubular apoptosis and autophagy. Life Sciences 2010;86:115-23.

41. Tandara AA, Kloeters 0, Kim I, et al. Age effect on HSP70: decreased resistance to ischemic and oxidative stress in HDF. $\mathrm{J}$ Surg Res 2006;132:32-9.

42. Moon H, Baek D, Lee B, et al. Soybean ascorbate peroxidase suppresses Baxinduced apoptosis in yeast by inhibiting oxygen radical generation, Bioch Biophys Res Comm 2002;290:457-62.

43. Iyer NV, Kotch LE, Agani F, et al. Cellular and developmental control of 02 homeostasis by hypoxia-inducible factor $1 \alpha$. Genes Dev 1998;12:149-62.

44. Thomopoulos GN, Spicer SS, Gratton MA, Schulte BA. Age-related thickening of basement membrane in stria vascularis capillaries. Hear Res 1997;111:31-41.

45. Rius J, Guma M, Schachtrup C, et al. NF-kB links innate immunity to the hypoxic response through transcriptional regulation of HIF-1 $\alpha$. Nature 2008;453:807-11.

46. Moreau C, Devos D, Brunaud-Danel V, et al. Elevated levels of IL-6 in CSF from sporadic ALS patients: inflammation or hypoxia? Neurology 2005;65:1958-60.

47. Vangeison G, Carr D, Federoff HJ, Rempe DA. The good, the bad and the cell typespecific roles of hypoxia inducible factor-1 alpha in neurons and astrocytes. J Neurosci 2008;28:1988-93. 